\title{
Hyperglycemia induces osteogenic differentiation of bone marrow derived stem cells: an in vitro study
}

\author{
Alessio Giai Via ${ }^{1}$ \\ Mary Beth McCarthy² \\ Michael Francke ${ }^{2}$ \\ Francesco Oliva ${ }^{3}$ \\ Augustus D. Mazzocca ${ }^{2}$ \\ Nicola Maffulli 4,5
}

1 Department of Orthopaedic Surgery and Traumatology, Hip surgery centre, IRCCS Policlinico San Donato, San Donato Milanese, Milan, Italy

2 Department of Orthopaedic Surgery, University of Connecticut, Farmington, Connecticut, USA

3 Department of Orthopaedic Surgery and Traumatology, University of Rome School of Medicine, Rome, Italy

${ }^{4}$ Department of Musculoskeletal Disorders, School of Medicine and Surgery, University of Salerno, Salerno, Italy

5 Queen Mary University of London, Barts and the London School of Medicine and Dentistry, Centre for Sports and Exercise Medicine, Mile End Hospital, London, England

Corresponding author:

Nicola Maffulli

Department of Musculoskeletal Disorders

School of Medicine and Surgery

University of Salerno, Salerno, Italy

Mary University of London

Barts and the London School of Medicine and

Dentistry

Centre for Sports and Exercise Medicine

Mile End Hospital 275 Bancroft Road

E1 4DG, London, England

Tel.: +447989358279

E-mail: n.maffulli@qmul.ac.uk

\section{Summary}

Introduction: The physiopathology of calcific tendinopathy (CT) is largely unknown. It could be the result of an active cell-mediated process. Many endocrine and metabolic diseases may impair the homeostasis of the tendon. The present study investigated whether hyperglycemia may influence the differentiation of bone marrow MSCs (bMSCs). The hypothesis is that high glucose levels may induce bone differentiation of MSCs.

Methods: Bone marrow (BM) was aspirated from the humeral head of three patients and concentrated. bMSCs were taken to the lab, counted, plated and grown to confluence. After $24 \mathrm{hrs}$ cells were treated with MEM supplemented with low (5.0 mM), physiological (10 $\mathrm{m} \mathrm{M})$ and high (25 mM) glucose, (+) and (-) $10 \mathrm{~m} \mathrm{M}$ insulin. Control cells were treated with MEM alone. Quantitative polymerase chain reaction (qPCR) was used to measure changes in gene expression levels specific for fibrocartilage in bMSCs.

Results: After 7 days, a significantly increased gene expression of collagen type I, type II, alkaline phosphatase and osteopontin was found in bMSCs supplemented with high glucose compared both to control group, and to low and physiological glucose groups with and without insulin $(p<0.05)$. When insulin was added to high glucose culture, a significantly higher expression of aggrecan, alkaline phosphatase, type I and II collagen, and fibronectin was found compared to all the other groups $(p<0.05)$.

Conclusion: When cultured in a high glucose medium, bMSCs express bone markers, and are able to differentiate toward an osteoblast lineage. CT may be caused by erroneous differentiation of MSCs in presence of high glucose serum levels.

KEY WORDS: calcific tendinopathy, tendons, mesenchymal stem cells, hyperglycemia, diabetes mellitus.

\section{Introduction}

Calcific tendinopathy (CT) is characterized by calcific deposits in the extracellular matrix (ECM) of the tendons. The physiopathology is still unknown, but rather than caused by precipitation of inorganic ions, it seems the result of an active cell-mediated process ${ }^{1}$. The ectopic bone formation may be caused by the erroneous differentiation of resident mesenchymal stem cells (MSCs) ${ }^{2-4}$, but it is a question that remains unsolved. In this study we will refer to insertional CT (bonny spur). 
There is increasing evidence about the influence of hormones and metabolic diseases, like hypothyroidism, obesity and diabetes mellitus, on tendons homeostasis ${ }^{5-7}$. More than $30 \%$ of patients with insulin-dependent diabetes have tendon calcifications. Snedeker and Gautieri have recently elucidated the relationship between diabetes mellitus and the changes of the tendon $\mathrm{ECM}^{8}$.

The purpose of this study is to investigate whether hyperglycemia may influence the differentiation of bone marrow MSCs (bMSCs). The hypothesis is that high dose glucose will negatively affect the gene expression levels specific for fibrocartilage of human MSCs.

\section{Materials and methods}

\section{Bone marrow aspiration and bMSC characterization}

Bone marrow (BM) was aspirated from the proximal humerus of 3 male patients during arthroscopic RC repair9 (Institutional Review Board \# 06577-2). BM was processed using an automated system (Angel, Arthrex Inc., Naples Fla.) with a $15 \%$ hematocrit setting. Cells were brought to the laboratory for counting and were plated for expansion at a concentration of $6 \times 10^{6}$ cells $/ 9.6 \mathrm{~cm}^{2}$ in complete medium containing minimum essential media (MEM) (Thermo Fisher Scientific, Waltham, MA), 10\% fetal bovine serum (Thermo Fisher Scientific) and 1\% penicillin/streptomycin (Thermo Fisher Scientific) in Primaria culture dishes (Thermo Fisher Scientific). After $24 \mathrm{hrs}$ in culture, medium was aspirated to remove non-adherent cells. Colony forming units (CFU) were counted after 10 days and cells were grown to confluence and expanded.

\section{FACS analysis}

To ensure a positive phenotype for MSCs, FACS analysis was employed. Confluent cells were trypsinized in $0.25 \%$ trypsin/EDTA, rinsed and centrifuged. The resulting pellet was resuspended in phosphate buffered saline (PBS) containing $1 \%$ human and fetal bovine serums and $1 \%$ bovine serum albumin. Cells were incubated with either phycoerythrin (PE) isothiocyanate or fluorescein antibodies (BD Bioscience, San Jose, CA) and analyzed using a FACS Calibur. To positively identify adult human stem cells, PE conjugated mouse monoclonal antiCD73 immunoglobulin (lgG), anti-CD90 lgG, and fluorescein isothiocyanate-conjugated anti-CD45 monoclonal IgG (BD Bioscience) were used ${ }^{10}$.

\section{Experimental design}

For all experiments, passage-1, bMSCs were plated onto 24 well Primaria tissue culture plates for $24 \mathrm{hrs}$ at a concentration of 40,00 cells $/ \mathrm{cm}^{2}$ containing glucose free MEM (Fig. 1). After 24 hrs, cells were treated with complete MEM supplemented with low (5 $\mathrm{mM})$, physiological $(10 \mathrm{mM})$ and high $(25 \mathrm{mM})$ glucose (Sigma, St Louis, MO), (+) and (-) 10-10 $\mathrm{mM}$ in- sulin (Sigma) for 0 , and $24 \mathrm{hrs}, 7$ and 14 days. The calcium content was measured after 4 weeks. Control cells were treated with complete MEM alone, with 1 $\mathrm{mM}$ glucose to sustain cells. All experiments were performed $3 X$ using bMSCs obtained from 3 different patients.

\section{Quantitative polymerase chain reaction ( $q P C R$ )}

Type I and type II collagen, alkaline phosphatase, fibronectin, osteopontin and aggrecan were chosen to indicate the ability of bMSCs to express genes associated with CT. RNA was isolated from cells using the TRIzol reagent (Thermo Fisher Scientific). Quantity and purity of the RNA were measured using a NanoDrop (Thermo Fisher Scientific). RNA was reverse transcribed into cDNA using $1 \mu \mathrm{g}$ messenger RNA and a High Capacity Reverse Transcription kit (Thermo Fisher Scientific). The qPCR was performed using 10-100 ng cDNA as a template, in triplicate for accuracy, using the StepOne Real-Time PCR System (Applied BioSystems, Foster City, California). Glyceradehyde 3-phosphate dehydrogenase (GAPDH) was used as the endogenous control for all samples. Three qPCR assays were performed for each of 3 patients with 3 replicates/experimental condition.

\section{Calcium and DNA content}

After 4 weeks, calcium was extracted twice for $30 \mathrm{~min}$ from the cells using 5\% TCA. Calcium content was measured in the cell extract using a calcium kit (Sigma, St Louis, MO). Mineralization was normalized to DNA content using fluorometric analysis. Cells were first disrupted by adding $0.01 \%$ SDS to each well for $15 \mathrm{~min}, 100 \mathrm{ml}$ of cell lysate was transferred to a clean 96 well plate and $100 \mathrm{ml}$ of filtered $10 \mathrm{mg} / \mathrm{ml}$ Hoechst dye was added to each well. DNA content was measured at $30-\mathrm{nm}$ excitation and $460-\mathrm{nm}$ emission using a microplate reader (Bio-TEK Synergy HT, Winooski, VT).

\section{Statistics}

Data are expressed as the mean \pm standard error or as $95 \%$ IC of the technical triplicates of 6-9 independent samples. Descriptive statistics are reported as mean and standard deviation. The Kruskal-Wallis test was used for all comparisons. The Wilcoxon ranksum test was used for pairwise comparisons of calcium content and the Dunn's test for companions of gene expression data $(p<0.05)$. All analyses were performed with Stata 12 (StataCorp. 2011. Stata Statistical Software: Release 12. College Station, TX: StataCorp LP).

\section{Results}

Between 60-160 ml's (mean 99.0 \pm 52.3 ) of BM was successfully aspirated from the patients (mean age: 56.4.2 \pm 7.4) and was concentrated to be used as a source of bMSCs (mean volume: $3.75 \pm 0.75 \mathrm{cc}$ 's). 

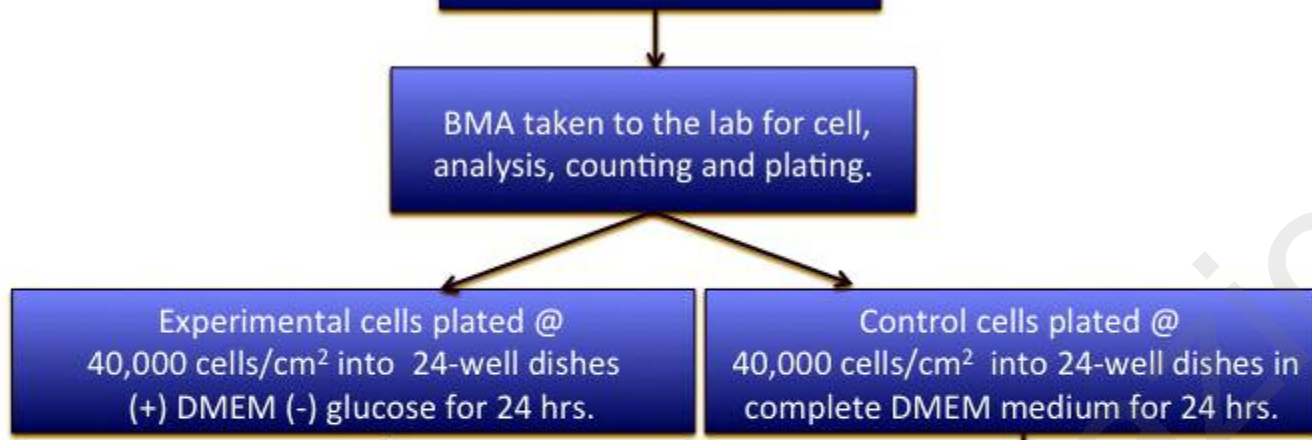

Medium aspirated after $24 \mathrm{hrs}$. and new

DMEM medium added to each well

containing Low, Physiological, and High

Glucose $(+) /(-)$ insulin

RNA extracted from cells for qPCR at $0,24,7$ and 14 days.

Medium collected for Calcium Content and DNA assays at 4 weeks

Figure 1. Schematic of study design and groups. Low $(5 \mathrm{mM})$, physiological $(10 \mathrm{mM})$ and high $(25 \mathrm{mM})$ glucose $(+)$ and $(-)$ 10-10 mM insulin. BM, bone marrow; BMA, bone marrow aspirate; qPCR, quantitative real-time polymerase chain reaction.

\section{bMSCs characterization by colony forming unit assay and FACS analysis}

A CFU assay was performed to determine whether the aspirated cells possess properties indicative of MSCs such as clonogenicity and multi-potency. We found that the cells were able to give rise to variablesized colonies after 10 days in culture. CFU's were counted after 10 days in culture to evaluate the number of bMSCs; $20.16 \pm 15.3$ CFU's grew $/ 10^{6}$ nucleated cells resulting in $1842 \pm 1287 \mathrm{bMSCs} / \mathrm{ml}$ of concentrated BM. Cells expressed a comprehensive set of surface markers to be found on adult human MSCs: CD73, CD90, and CD105 but were negative for hematopoietic surface marker CD45 ${ }^{11}$.

\section{Gene expression}

Type I collagen and fibronectin gene expression was significantly increased at $24 \mathrm{hrs}$ and 7 days in the high glucose (HG) group compared to other groups $(p<0.05$ and 0.001$)$. When insulin was added to the $\mathrm{MEM}$, an increase in type I collagen and fibronectin expression was observed at the 14 day in the $\mathrm{HG}$ group $(p<0.05)$ (Fig. 2A and D).

After 24 hrs and 7 days, type II collagen gene expression was increased in bMSCs supplemented with HG compared to control, low and physiological groups and to the physiological and $\mathrm{HG}$ groups (+) insulin $(p<0.05)$. By 14 days, the gene expression was in- creased when insulin was added to the low and HG cultures compared to other groups $(p<0.05)$ (Fig. 2B). The HG group (+) insulin at 7 and 14 days had higher aggrecan expression compared to the low, physiological and HG groups, and the low and physiological (+) insulin groups $(p<0.05)$. The $H G(+)$ insulin group, at 7 days, had the highest expression at all other time points $(p<0.001)$. Interestingly, the physiological group also had significantly high expression at 7 days $(p<0.001)$ but was down regulated at the 14 day time point (Fig. 2C).

There was higher osteopontin expression in the HG group compared to the control $(p<0.001)$, low and physiological groups at $24 \mathrm{hrs}, 7$ and 14 days $(p<0.05)$. At 14 days gene expression was increased in the low and HG group (+) insulin ( $p<0.05)$ (Fig. 2E).

There was a higher alkaline phosphatase gene expression after $24 \mathrm{hrs}$ and 7 days in the HG group $(p<0.05)$. At the 14 day higher gene expression was seen in the $\mathrm{HG}(+)$ insulin group compared to all other groups $(p<0.001)$ (Fig. $2 F)$.

\section{Calcium content}

HG with or without insulin significantly stimulated the amount of calcium per mg DNA in BM-MSCs cultures compared to control, low and physiological groups (+) and $(-)$ insulin $(p<0.001)$. No significant differences were found between the other groups (Fig. 3). 


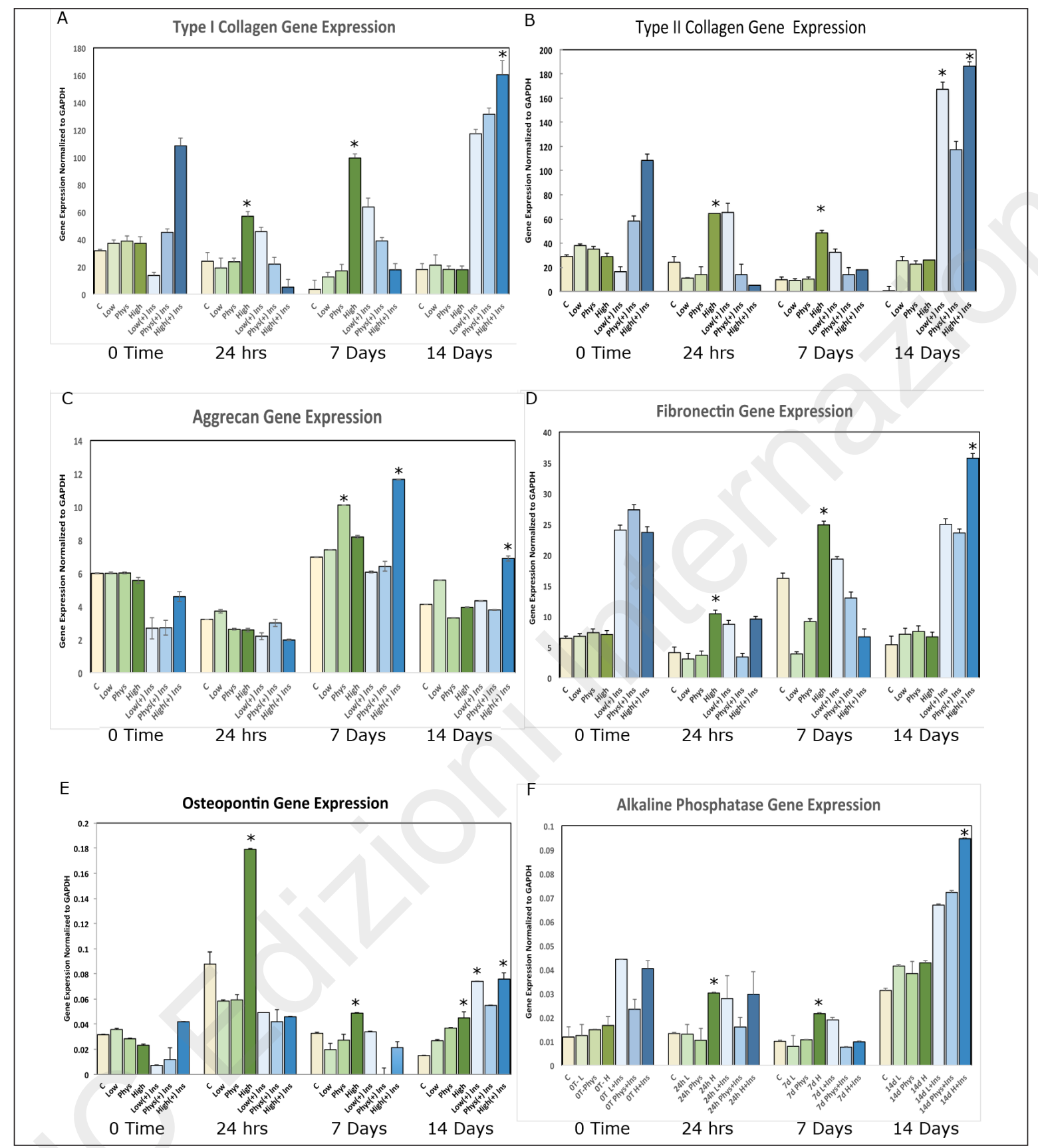

Figure 2. There was a significant increase in type I collagen and fibronectin expression at the 14 days time point compared to all other times and groups when insulin was added to the high glucose group $(A, D)(p<0.05)$. At 24 hrs and 7 days type I collagen and fibronectin gene expression was significantly increase in the high glucose group compared to all other group within their times point $(p<0.05$ and 0.001 respectively). bMSCs supplemented with high glucose had a significant increase in type II collagen after $24 \mathrm{hrs}$. and 7 days compared to control, low and physiological groups and to physiological and high glucose groups $(+)$ insulin $(B)(p<0.05)$. When insulin was added to the low and high glucose cultures at 14 days, a significant increase in type II collagen was seen compared to all other groups at the same time point $(p<0.05)(B)$. C shows that the gene expression for aggrecan was significantly increased in the high glucose group (+) insulin at 7 and 14 days compared to the low, physiological and high glucose group and the low and physiological $(+)$ insulin groups $(p<0.05)$. Osteopontin gene expression was significantly higher in the high glucose group at $24 \mathrm{hrs}$. and 7 days compared to the low and physiological groups $(p<0.05)$. When insulin was added to the low and high glucose groups at 14 days, there was a significant increase in osteopontin compared to all other groups at the same time point $(p<0.05)(E)$. $F$ shows that alkaline phosphatase gene expression was significantly increase at $24 \mathrm{hrs}$ and 7 days in the high glucose groups compared to low, and physiological glucose groups $(p<0.05)$. Gene expression was increased when insulin was added to the high glucose group at 14 days compared to all other groups at all time points $(p<0.001)$. 


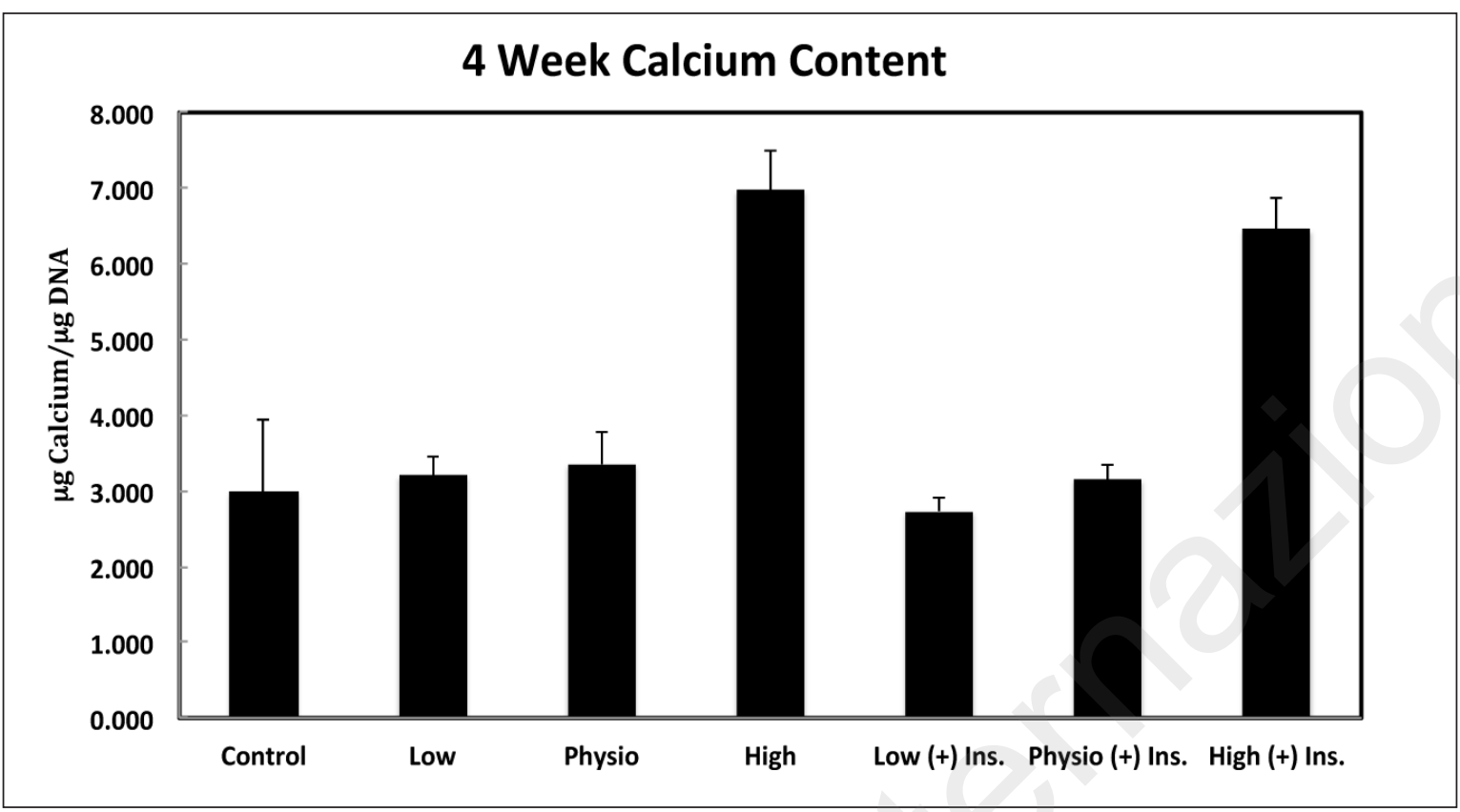

Figure 3. Effect of different concentrations of glucose (+) or (-) insulin at 4 weeks on mineralization. The amount of calcium per mg DNA was significantly increased in the high glucose group (+) or (-) insulin after 4 weeks in culture.

\section{Discussion}

The aetiopathogenesis of CT is controversial, but it seems the result of an active cell-mediated process. Animal studies showed that insertional Achilles CT is generated by a process similar to endochondral ossification, and it has a structure similar to trabecular bone ${ }^{12}$. In this process, resident MSCs and their erroneous differentiation into osteoblasts may play an important role ${ }^{5}$. Several studies discussed the adverse effects of MSCs in pathogenic conditions, including vascular calcification ${ }^{13}$, aortic valve calcification ${ }^{14}$, fibrodysplasia ossificans progressive ${ }^{11}$. Recently the role of MSCs has been studied in the ossification process of the posterior longitudinal ligament of the spine $^{15}$, but the mechanism leading to the differentiation of MSCs is still unknown.

The microenvironment may play a key role. Stem cells are influenced by their microenvironment. They respond to a variety of signals which most originate from the ECM and are able to regulate stem cell behaviors, proliferation, self-renewal and differentiation ${ }^{16}$. At the same time, MSCs secrete different agents that are able to modify the characteristics of the ECM and the differentiation signals ${ }^{17,}{ }^{18}$. This means that MSCs and ECM are in constant connection to each other. It is easy to understand that local modifications of the ECM are able to affect the differentiation of $\mathrm{MSCs}^{19}$.

The alterations of EMC in tendinopathy are well known ${ }^{20}$. But why MSCs should differentiate into an osteoblast lineage? Liu et al. ${ }^{21}$ studied the effect of HG levels on human aortic smooth muscle cells in vitro. $\mathrm{HG}$ levels increase the expressions of BMP-2 and
Cbfa-1, alkaline phosphatase activity, and intracellular calcium deposition, concluding that HG levels can induce the calcification of vascular smooth muscle cells by inducing osteoblastic differentiation and intracellular calcium deposition. Akune et al. ${ }^{22}$ examined the relationship between diabetes mellitus and the extent of ossification in patients with ossification of the posterior longitudinal ligament. They found that the insulin secretory response was strongly associated with the extent of ossification. Furthermore, insulin is known to induce a wide variety of growth and metabolic responses, and to play important roles in the anabolic regulation of bone metabolism ${ }^{23,24}$. Insulin induces a cellular response by binding both to its own endogenous receptor (IRS-1), and to insulin-like growth factor-I (IGF-I) receptor, which is an anabolic factor for bone formation ${ }^{25}$. Some studies showed the relationship between insulin and heterotopic calcifications. In vitro, high dose of insulin accelerates the calcification in human vascular smooth muscle cells ${ }^{26,27}$. Furthermore, one of the most important features of diabetes mellitus is the insulin resistance, and higher levels of insulin are usually detected in type II diabetic patients ${ }^{28}$. It is therefore reasonable that high glucose levels and insulin may act as a mediators of CT in patients with diabetes mellitus.

Type I collagen is the most abundant type of collagen in the human body. An increase in type I collagen may account for poor ECM organization ${ }^{29}$. Type II collagen is an important component of the ECM at the $\mathrm{TBJ}^{30}$, and the expression of type II collagen is upregulated in human $\mathrm{RC}$ tendinopathy $\mathrm{y}^{7,31}$. The $\mathrm{mR}$ NA and protein expression of aggrecan, and its relationship with ectopic ossification, has been observed 
in a CT model 29 . An interesting histological study on rotator cuff CT found an increased expression of osteopontin in the calcific area of the supraspinatus tendons compared to healthy tendon ${ }^{32}$. Osteopontin is a protein produced by osteoblasts which is involved in anchoring the osteoclasts to the mineral of bone matrix, it has an important role in matrix remodeling, and it is upregulated during chondrocyte terminal differentiation $^{33}$. Therefore, it could play an important role during the formation of the calcific depositions. Alkaline phosphatase is produced during mineralization in the precalcific stage ${ }^{34}$. To support our theory, we found a statistically significant increased expression of collagen type I and II, aggrecan, fibronectin, osteopontin and ALK in bMSCs when cultured with HG compared to low and physiological glucose concentrations. Furthermore, when insulin was added to the medium a significantly higher expression of bone markers was found compared to cells cultured without insulin. These data support our theory that insertional CT may be caused by the erroneous differentiation of resident bMSCs in presence of HG levels.

Our experiments have some limitations. The differentiation of MSCs may be influenced by donor behaviors, the culture media, and the passage proliferation. Even if the donor sample was not uniform, the cell were selected and expanded in the same way, suggesting that the culture conditions were a significant variable on MSCs differentiation. Then, we used MSCs harvested from the bone marrow for our experiment. This allowed us to speculate only on the pathogenesis of insertional CT.

The study has been performed according the basic principles and recommendations in clinical and field science research ${ }^{35}$.

\section{Conclusion}

When cultured in a HG medium, bMSCs express bone markers, and are able to differentiate toward an osteoblast lineage. These results reinforce the concept that CT may be caused by erroneous differentiation of MSCs in the presence of high level of serum glucose.

\section{Conflict of interest}

The Authors declare no financial support or other benefits from commercial sources for the work reported on in the manuscript, nor any other financial interests which could create a potential conflict of interest or the appearance of a conflict of interest with regard to the work.

\section{References}

1. Oliva F, Via AG, Maffulli N. Physiopathology of intratendinous calcific deposition. BMC Med. 2012;23;10:95.
2. Rui YF, Lui PP, Ni M, Chan LS, Lee YW, Chan KM. Mechanical loading increased BMP-2 expression which promoted osteogenic differentiation of tendon-derived stem cells. J Orthop Res. 2010;29:390-396.

3. Rui YF, Lui PP, Chan LS, Chan KM, Fu SC, Li G. Does erroneous differentiation of tendon-derived stem cells contribute to the pathogenesis of calcifying tendinopathy? Chin Med J. 2011;124:606-610.

4. Zhang J, Wang JH. BMP-2 mediates PGE(2)-induced reduction of proliferation and osteogenic differentiation of human tendon stem cells. J Orthop Res. 2012;30:47-52.

5. Oliva F, Piccirilli E, Berardi AC, Tarantino U, Maffulli N. Influence of Thyroid Hormones on Tendon Homeostasis. Adv Exp Med Biol. 2016;920:133-138.

6. Oliva F, Piccirilli E, Berardi AC, Frizziero A, Tarantino U, Maffulli N. Hormones and tendinopathies: the current evidence. $\mathrm{Br}$ Med Bull. 2016;117:39-58.

7. Zreik NH, Malik RA, Charalambous CP. Adhesive capsulitis of the shoulder and diabetes: a meta-analysis of prevalence. Muscles Ligaments Tendons J. 2016;6:26-34. Review.

8. Snedeker JG, Gautieri A. The role of collagen crosslinks in ageing and diabetes - the good, the bad, and the ugly. Muscles Ligaments Tendons J. 2014;17:303-308.

9. Beitzel K, McCarthy MB, Cote MP, et al. Comparison of mesenchymal stem cells (osteoprogenitors) harvested from proximal humerus and distal femur during arthroscopic surgery. Arthroscopy. 2013;29:301-308.

10. Dominici M, Le Blanc K, Mueller I, et al. Minimal criteria for defining multipotent mesenchymal stromal cells. The International Society for Cellular Therapy position statement. Cytotherapy. 2006;8:315-317.

11. Medici D, Shore EM, Lounev VY, et al. Conversion of vascular endothelial cells into multipotent stem-like cells, Nat. Med. 2010;16:1400-1406.

12. Lui PP, Fu SC, Chan LS, Hung LK, Chan KM. Chondrocyte phenotype and ectopic ossification in collagenase-induced tendon degeneration. J Histochem Cytochem. 2009;57:91-100.

13. Tang R, Gao M, Wu M, Liu H, Zhang X, Liu B. High glucose mediates endothelial-to-chondrocyte transition in human aortic endothelial cells. Cardiovasc Diabetol. 2012;22:113.

14. Chen JH, Yip CY, Sone ED, et al. Identification and characterization of aortic valve mesenchymal progenitor cells with robust osteogenic calcification potential. Am J Pathol. 2009;174: 1109-1119.

15. Harada $Y$, Furukawa K, Asari T, et al. Osteogenic lineage commitment of mesenchymal stem cells from patients with ossification of the posterior longitudinal ligament. Biochem Biophys Res Commun. 2014;17:1014-1020.

16. Yang G, Rothrauff BB, Lin H, Yu S, Tuan R. Tendon-Derived Extracellular Matrix Enhances TGF- $\beta 3$ Induced Tenogenic Differentiation of Human Adipose-Derived Stem Cells. Tissue Eng Part A. 2016 Nov 3. [Epub ahead of print]

17. Mardones R, Giai Via A, Jofré C, Minguell J, Rodriguez C, Tomic A, Salineros M. Cell therapy for cartilage defects of the hip. Muscles, Ligaments and Tendons J. 2016;6:361-366.

18. Lozito TP, Taboas JM, Kuo CK, Tuan RS. Mesenchymal stem cell modification of endothelial matrix regulates their vascular differentiation. J Cell Biochem. 2009;107:706-713.

19. Hagmann S, Moradi B, Frank S, Dreher T, Kämmerer PW, Richter W, Gotterbarm T. Different culture media affect growth characteristics, surface marker distribution and chondrogenic differentiation of human bone marrow-derived mesenchymal stromal cells. BMC Musculoskelet Disord. 2013;30:223.

20. Giai Via A, Papa G, Oliva F, Maffulli N. Tendinopathy. Curr Phys Med Rehabil Rep. 2016;4:50-55.

21. Liu F, Zhong H, Liang JY, et al. Effect of high glucose levels on the calcification of vascular smooth muscle cells by inducing osteoblastic differentiation and intracellular calcium deposition 
via BMP-2/Cbfa-1 pathway. J Zhejiang Univ Sci B. 2010;11: 905-911.

22. Akune T, Ogata N, Seichi Ai, Ohnishi I, Nakamura K, Kawaguchi H. Insulin Secretory Response Is Positively Associated with the Extent of Ossification of the Posterior Longitudinal Ligament of the Spine. JBJS. 2001;83:1537-1544.

23. Thomas DM, Hards DK, Rogers SD, Ng KW, Best JD. Insulin receptor expression in bone. J Bone Miner Res. 1996;11: 1312-1320.

24. Mazzocca AD, McCarthy MB, Chowaniec D, Cote MP, Judson $\mathrm{CH}$, Apostolakos J, et al. Bone marrow-derived mesenchyma stem cells obtained during arthroscopic rotator cuff repair surgery show potential for tendon cell differentiation after treatment with insulin. Arthroscopy. 2011;27:1459-1471.

25. Canalis $\mathrm{E}$. Insulin like growth factors and the local regulation of bone formation. Bone. 1993;14:273-276.

26. Yano $\mathrm{H}$, Ohya $\mathrm{K}$, Amagasa $\mathrm{T}$. Effects of insulin on in vitro bone formation in fetal rat parietal bone. Endocr J. 1994;41:293-300.

27. Olesen P, Nguyen K, Wogensen L, et al. Calcification of human vascular smooth muscle cells: associations with osteoprotegerin expression and acceleration by high-dose insulin. Am J Physiol Heart Circ Physiol. 2007;292:1058-1064.

28. Weyer C, Funahashi T, Tanaka S, et al. Hypoadiponectinemia in Obesity and Type 2 Diabetes: Close Association with Insulin Resistance and Hyperinsulinemia. J Clin Endocrinol Metab. 2001;86:1930-1935.
29. Lui PP, Chan LS, Lee W, Fu SC, Chan KM. Sustained expression of proteoglycans and collagen type III/type I ratio in a calcified tendinopathy model. Rheumatology (Oxford). 2010;49: 231-239.

30. Apostolakos J, Durant TJ, Dwyer CR, et al. The enthesis: a review of the tendon-to-bone insertion. Muscles Ligaments Tendons J. 2014 17:4:333-342.

31. Sharma P, Mafffulli N. Biology of tendon injury: healing, modeling and remodeling. J Musculoskelet Neuronal Interact. 2006;6:181-190.

32. Oliva F, Barisani D, Grasso A, Maffulli N. Gene expression analysis in calcific tendinopathy of the rotator cuff. Eur Cell Mater. 2011 Jun 20;21:548-557.

33. Reinholt FP, Hultenby K, Heinegard D, Marks SC Jr, Norgard M, Anderson G. Extensive clear zone and defective ruffled border formation in osteoclasts of osteopetrotic (ia/ia) rats: implication for secretory function. Exp Cell Res. 1999; 15:477-491.

34. O'Brien EJ, Frank CB, Shrive NG, Hallgrìmsson B, Hart DA Heterotopic mineralization (ossification or calcification) in tendinopathy or following surgical tendon trauma. Int J Exp Pathol. 2012;93:319-331.

35. Padulo J, Oliva F, Frizziero A, Maffulli N. Muscles, Ligaments and Tendons Journal - Basic principles and recommendations in clinical and field science research: 2016 update. MLTJ 2016;6(1):1-5. 